\title{
Venous Thromboembolism Due to Suspected Interaction Between Rivaroxaban and Nevirapine
}

\author{
Duane Bates, Bruce Dalton, Janet Gilmour, and Jeff Kapler
}

\section{INTRODUCTION}

$\mathrm{R}$ ivaroxaban is a selective, reversible inhibitor of factor Xa that is used for the prevention and treatment of venous thromboembolism (VTE) and for the prevention of stroke in patients with atrial fibrillation. Nevirapine is a non-nucleoside reverse transcriptase inhibitor that is generally given in combination with 2 nucleoside reverse transcriptase inhibitors to suppress HIV in infected individuals. Both medications are metabolized in the liver through the cytochrome (CYP) P450 pathway. Nevirapine is also an enzyme inducer that may increase the clearance of rivaroxaban. There is a lack of information about drug interactions involving rivaroxaban after total joint replacement surgery. ${ }^{1}$ We report a case of VTE after total knee replacement that may have been due to an interaction between rivaroxaban and nevirapine.

\section{CASE REPORT}

A 60-year-old man was admitted to hospital for total knee replacement.* The patient's medical history included hypertension, dyslipidemia, cerebrovascular accident of the right middle cerebral artery, osteoarthritis, post-herpetic neuralgia and polyneuropathy, and HIV infection. The HIV infection had been diagnosed 8 years before the current admission (viral load 270000 copies $/ \mathrm{mL}$; CD4 nadir $0.266 \times 10^{9} / \mathrm{L}$ [normal range $0.499 \times 10^{9}$ to $\left.\left.1.651 \times 10^{9} / \mathrm{L}\right]\right)$, but the patient had never had an AIDS-defining illness. The patient had no prior history of VTE and no known drug allergies. His body weight was $61.1 \mathrm{~kg}$.

Medications before admission were nevirapine $400 \mathrm{mg}$ daily, lamivudine $150 \mathrm{mg}$ twice daily, zidovudine $300 \mathrm{mg}$ twice

\footnotetext{
${ }^{*}$ The patient gave verbal and written consent to publish this case report.
}

daily, amlodipine $10 \mathrm{mg}$ daily, metoprolol $50 \mathrm{mg}$ twice daily, lisinopril $20 \mathrm{mg}$ daily, hydrochlorothiazide $25 \mathrm{mg}$ daily, atorvastatin $20 \mathrm{mg}$ daily, acetylsalicylic acid $81 \mathrm{mg}$ daily, celecoxib $200 \mathrm{mg}$ daily, omeprazole $20 \mathrm{mg}$ daily, gabapentin $300 \mathrm{mg} 3$ times daily, and lamotrigine $50 \mathrm{mg}$ twice daily. The only change to the medication regimen in the past 2 years had been the discontinuation, about 3 weeks before admission, of abacavir $600 \mathrm{mg} /$ lamivudine $300 \mathrm{mg}$ because of intolerance and replacement with lamivudine $150 \mathrm{mg} /$ zidovudine $300 \mathrm{mg}$. At the time of the change in antiretroviral therapy, the patient had a viral load of less than 40 copies $/ \mathrm{mL}$ and a CD4 count of $0.392 \times 10^{9} / \mathrm{L}$, which suggested a good virological response to therapy.

The patient received dalteparin 2500 units subcutaneously (SC) $6 \mathrm{~h}$ after the knee replacement surgery and then dalteparin 5000 units SC on postoperative days 1 and 2. Rivaroxaban $10 \mathrm{mg}$ daily was started on postoperative day 3 , at which time the patient was discharged from hospital; there were no other changes to the patient's home medication regimen. At the time of discharge, the patient had no symptoms of VTE.

On postoperative day 4 (after 2 doses of rivaroxaban) the patient presented to the emergency department with shortness of breath, chest pain, and swelling of the left leg. He was hemodynamically stable, with oxygen saturation of $93 \%$ on room air and temperature $38.3^{\circ} \mathrm{C}$. The only abnormalities noted on physical examination were a grade $2 / 6$ systolic murmur and jugular venous pressure of $1-2 \mathrm{~cm}$ above the sternal angle. The left leg was warm and swollen, but no erythema or discharge was noted around the surgical site.

The results of laboratory tests at the time of this admission included hemoglobin $100 \mathrm{~g} / \mathrm{L}$ (normal range 137-180 g/L), platelet count $139 \times 10^{9} / \mathrm{L}$ (normal range $150 \times 10^{\%} / \mathrm{L}$ to $400 \times 10^{\%} / \mathrm{L}$ ), white blood cell count $4.9 \times 10^{\%} / \mathrm{L}$ (normal range $4 \times 10^{9} / \mathrm{L}$ to $11 \times 10^{\%} / \mathrm{L}$ ), international normalized ratio (INR) 1 (normal range 0.9-1.1), activated partial thromboplastin 
time $25.4 \mathrm{~s}$ (normal range 25-35 s), serum creatinine $107 \mu \mathrm{mol} / \mathrm{L}$ (normal range $50-120 \mu \mathrm{mol} / \mathrm{L}$ ), and an estimated creatinine clearance of $57 \mathrm{~mL} / \mathrm{min}$ (according to the Cockcroft-Gault formula). The results of liver function tests were total bilirubin $8 \mu \mathrm{mol} / \mathrm{L}$ (normal range 0-24 $\mu \mathrm{mol} / \mathrm{L}$ ), alanine aminotransferase $74 \mathrm{U} / \mathrm{L}$ (normal range 1-60 U/L), alkaline phosphatase $74 \mathrm{U} / \mathrm{L}$ (normal range 30-145 U/L), and $\gamma$-glutamyl transferase $15 \mathrm{U} / \mathrm{L}$ (normal range $11-63 \mathrm{U} / \mathrm{L}$ ). Elevation of D-dimer $(3.44 \mathrm{mg} / \mathrm{L} \mathrm{FEU}$ [fibrinogen equivalent units]; normal range $\leq 0.46 \mathrm{mg} / \mathrm{L} \mathrm{FEU}$ ) and troponin $\mathrm{T}$ (21 ng/L; normal range 1-14 ng/L) were noted. Contrastenhanced computed tomography of the lower legs and chest revealed a left-sided deep vein thrombosis (DVT) in the external iliac and common femoral veins, but the image was nondiagnostic for pulmonary embolism. There was no radiologic or clinical evidence of pneumonia. The results of blood, urine, and sputum culture were all negative. Electrocardiography displayed sinus tachycardia and lateral ST-T wave abnormalities.

In the emergency department, oxygen was started at $3 \mathrm{~L} / \mathrm{min}$, along with unfractionated heparin (80 unit/kg bolus, followed by infusion of 18 units/kg per hour) and warfarin. The rivaroxaban was stopped, and all other home medications were continued. On postoperative day 5, the heparin was switched to tinzaparin 10700 units (175 units/kg) SC daily; at this point, the chest pain had resolved. On postoperative day 7 , the oxygen was discontinued. As well, the only abnormality seen on transthoracic echocardiography was trace mitral regurgitation and trivial tricuspid regurgitation; the size and function of the left and right ventricles were normal. On postoperative day 9, the patient was discharged with prescriptions for warfarin (INR 3) and tinzaparin and was to be followed by Anticoagulation Management Services for INR monitoring. On postoperative day 10, the tinzaparin was discontinued (INR 2.5). On postoperative day 24 (INR 2.2), Anticoagulation Management Services transferred warfarin management to the family physician. The patient was stabilized with warfarin therapy as follows: $4 \mathrm{mg}$ on Monday, Wednesday, Friday, and Saturday and $5 \mathrm{mg}$ on Tuesday, Thursday, and Sunday.

\section{DISCUSSION}

Previous studies have shown that rivaroxaban is efficacious in preventing VTE after knee replacement surgery. Two randomized, double-blind studies, RECORD $3(n=2531$ patients) and RECORD 4 ( $n=3148$ patients), compared enoxaparin $40 \mathrm{mg}$ SC daily or enoxaparin $30 \mathrm{mg}$ SC twice daily with rivaroxaban $10 \mathrm{mg}$ daily after total knee replacement. ${ }^{2,3}$ In these trials, study medications were continued for 10-15 days, and patients were followed for 30-35 days. Mandatory bilateral venography was conducted between days 11 and 15. The primary outcome was a composite of DVT, nonfatal pulmonary embolism, or death from any cause during the treatment period. A modified intention-to-treat analysis was used in both studies and included patients who had undergone surgery, who took the study medication, and who had adequate assessment for thromboembolism. In the RECORD 3 trial, the modified intention-to-treat analysis ( $n=1702$ patients) revealed that the primary efficacy outcome occurred in $9.6 \%$ of the patients who received rivaroxaban and $18.9 \%$ of those who received enoxaparin $(p<0.001)$, which corresponded to an absolute risk reduction of $9.3 \%$ and a number needed to treat of $11 .^{2}$ In the RECORD 4 study, the modified intention-totreat analysis ( $n=1924$ patients) revealed that the primary efficacy outcome occurred in $6.9 \%$ of the patients taking rivaroxaban and $10.1 \%$ of those taking enoxaparin $(p=$ $0.0118)$, which corresponded to an absolute risk reduction of $3.2 \%$ and a number needed to treat of $31 .^{3}$ The incidence of major bleeding and non-major, clinically relevant bleeding was greatest in the rivaroxaban treatment arms, corresponding to an absolute risk increase of $0.64 \%$ and a number needed to harm of $156(p=0.039) .^{4}$

Unfortunately, the timing of VTE in the RECORD trials was not recorded, but venography was conducted between days 11 and $15.2,3$ The risk of VTE is highest shortly after surgery, and the median time of diagnosis for thromboembolic events is 7 days after total total knee replacement. ${ }^{5}$ The patient described in the current report presented with VTE 4 days after knee replacement surgery, and his VTE may have been related to an increased thromboembolic risk secondary to HIV infection and/or potentially enhanced clearance of rivaroxaban through a drug interaction with nevirapine.

A search of various drug interaction resources did not reveal any potential interactions between rivaroxaban and nevirapine..$^{6-11}$ A review of Embase (January 1980 to December 2012), OVID (January 1946 to December 2012), International Pharmaceutical Abstracts (January 1970 to December 2012), and Reactions Weekly (January 1990 to December 2012) using the search terms "rivaroxaban" and "nevirapine" yielded no citations. Lexi-Interact 2012 listed nevirapine as a strong inducer of isozyme CYP3A4, suggesting that nevirapine may decrease the serum concentration of rivaroxaban. ${ }^{12}$ The severity was listed as major, and the reliability rating was fair. The references for this interaction were the Canadian and US product monographs. The area under the curve and maximum serum concentration for rivaroxaban were approximately 50\% and $22 \%$ lower, respectively, when a single dose of the drug (20 mg) was administered to individuals who were also taking rifampin, a known strong inducer of isozyme CYP3A4 (titrated up to $600 \mathrm{mg} /$ day). ${ }^{12,13}$ A similar statement appeared on the University of Liverpool website for interactions involving HIV drugs. ${ }^{14}$ In contradiction, another drug interaction reference suggested that nevirapine is a weak inducer of isozyme CYP3A4. ${ }^{8}$ The Drug Interaction Probability scale developed by 
Horn and others ${ }^{15}$ suggests a possible drug interaction between nevirapine and rivaroxaban.

Treatment with drugs such as the rifamycins and some anticonvulsants (e.g., phenytoin, phenobarbital, carbamazepine) known to induce or up-regulate CYP enzymes predictably results in a marked reduction (by as much as 95\%) in the plasma concentration of certain drugs administered concurrently. ${ }^{16}$ Furthermore, the anticoagulant effect of rivaroxaban correlates with plasma concentration. ${ }^{13}$ CYP3A activity is particularly sensitive to such modulation, and the metabolism of CYP3A substrates is affected by enzyme-inducing agents. Generally, the consequences of CYP3A induction do not appear immediately, since new protein must be synthesized. Steady-state levels are generally reached within 2-3 weeks. ${ }^{16}$ Metabolic induction dissipates slowly over a period of days to weeks, depending on the half-life of the inducer, the half-life of the CYP isozyme, the presence of hepatic disease, and the patient's age. ${ }^{17}$ The patient described here had received nevirapine for years, so maximal enzyme induction of CYP3A4 was already present. The half-life of rivaroxaban is $5-13 \mathrm{~h}$, so the patient's plasma rivaroxaban levels potentially reached steady state within $48 \mathrm{~h}$ of starting rivaroxaban therapy.

Evaluation of the potential for an interaction between rivaroxaban and nevirapine relies on an understanding of the metabolism of the 2 drugs. Approximately two-thirds of rivaroxaban administered is metabolized to inactive metabolites and excreted in the urine and feces. The remaining third is excreted unchanged via the kidney. Approximately 30\% of administered drug is metabolized via hydrolysis and oxidative degeneration catalyzed by CYP P450 isozymes 3A4, 3A5, and 2J2. In addition, rivaroxaban is a substrate of P-glycoprotein. Drug interactions involving both P-glycoprotein and CYP3A4 inducers or inhibitors are likely to be more substantial than those affecting either system alone. ${ }^{1,13,18}$

Nevirapine undergoes extensive oxidative metabolism via the CYP system to several hydroxylated metabolites. The metabolism of nevirapine is mediated in part by the CYP3A4 and CYP2B6 isozymes; conversely, nevirapine also induces these isozymes by approximately $20 \%$ to $25 \%$, which results in autoinduction of its own metabolism. In addition, nevirapine is an inducer of drugs metabolized by these isozymes, with maximal effect on CYP3A4 within 2-4 weeks after initiation of therapy. Autoinduction also results in a corresponding decrease in the plasma half-life of nevirapine, from approximately $45 \mathrm{~h}$ with a single dose to approximately $25-30 \mathrm{~h}$ following multiple daily dosing in adults. ${ }^{12,19}$ Nevirapine does not inhibit P-glycoprotein.

No information was found about increasing the dose of rivaroxaban if an enzyme-inducing agent is used concurrently. Phase II dose-finding studies of rivaroxaban after major orthopedic surgery did not support a dose-response relationship for the primary end point of any DVT, nonfatal pulmonary embolism, or death from any cause. ${ }^{4}$ A randomized, doubleblind, double-dummy phase II dose-finding study $(n=621$ patients) of oral rivaroxaban $2.5 \mathrm{mg}, 5 \mathrm{mg}, 10 \mathrm{mg}, 20 \mathrm{mg}$, or $30 \mathrm{mg}$ twice daily versus enoxaparin $30 \mathrm{mg} S \mathrm{C}$ twice daily after total knee replacement showed a significant increase in major bleeding with increasing dose $(p=0.0007){ }^{20}$

One of the major dilemmas would be how best to monitor the effect of rivaroxaban, in order to balance the risks and the benefits, if the dose were increased for a patient receiving an enzyme-inducing medication. A chromogenic anti-Xa assay is the preferred test for monitoring rivaroxaban. ${ }^{21}$ In a quality control study, ${ }^{22}$ plasma samples were drawn from 20 healthy volunteers before and $2-3 \mathrm{~h}$ after a $10-\mathrm{mg}$ oral dose of rivaroxaban and were analyzed with a chromogenic anti-Xa assay in 9 Swiss laboratories. The mean accuracy was $7 \%$ and the mean coefficient of variation for precision of rivaroxaban quantification was $8.8 \% .{ }^{22}$ In randomized dose-ranging studies involving patients with normal renal function treated with approximately $22 \mathrm{mg}$ of rivaroxaban daily for DVT, the mean maximum serum concentration $\left(C_{\max }\right) 2-4 \mathrm{~h}$ after dosing was $270 \mu \mathrm{g} / \mathrm{L}(95 \%$ confidence interval [CI] approximately $160-400 \mu \mathrm{g} / \mathrm{L})$ and the mean trough concentration $\left(C_{\text {trough }}\right)$ was $25.5 \mu \mathrm{g} / \mathrm{L}(95 \% \mathrm{CI}$ approximately $10-200 \mu \mathrm{g} / \mathrm{L}) .{ }^{23}$ Rivaroxaban plasma concentrations associated with increased risk of bleeding are not currently available.

The 2012 clinical practice guidelines of the American College of Chest Physicians list many treatment options for VTE prophylaxis in patients undergoing total knee arthroplasty, suggesting 10 to 14 days of treatment with either lowmolecular-weight heparin, unfractionated heparin, fondaparinux, warfarin, apixaban, rivaroxaban, dabigatran, acetylsalicylic acid, or an intermittent pneumatic compression device. ${ }^{5}$ We are not aware of any clinicians using acetylsalicylic acid monotherapy to prevent VTE after orthopedic surgery. A lowmolecular-weight heparin, fondaparinux, or unfractionated heparin could have been used for the patient described here and would not have interacted with his antiretroviral therapy. The patient was initially started on dalteparin 5000 units SC daily, which could have been continued for 10-14 days instead of being switched to rivaroxaban.

Warfarin is problematic because it interacts with many antiretroviral medications, such as non-nucleoside reverse transcriptase inhibitors, protease inhibitors, and the integrase inhibitor elvitegravir. ${ }^{10,11,14}$ Furthermore, empiric warfarin dosage adjustments are challenging because of limited clinical evidence and lack of pharmacokinetic studies. ${ }^{24}$ Therefore, the effect on INR response should be used to guide warfarin dosage requirements in patients receiving antiretrovirals.

Apixaban and dabigatran would have been the 2 remaining options for VTE prophylaxis in the patient described in this 
case report. Approximately $15 \%$ of apixaban is metabolized via isozyme CYP3A4, compared with $30 \%$ for rivaroxaban. ${ }^{18}$ However, there is no information on the concurrent use of nevirapine and apixaban. The apixaban product monograph states that coadministration of apixaban with rifampin, a strong inducer of both isozyme CYPA4 and P-glycoprotein, led to decreases of approximately $54 \%$ in mean apixaban area under the curve and approximately $42 \%$ in maximum concentration. ${ }^{25}$ These results suggest the potential for reduction in serum concentrations of apixaban and efficacy if combined with nevirapine. Given the lack of data, this combination cannot be recommended.

Dabigatran might have been a therapeutic option, as there were no clinically significant CYP P450 or P-glycoprotein drug interactions with the patient's existing drug regimen. The patient's creatinine clearance was $57 \mathrm{~mL} / \mathrm{min}$, which suggested no risk of dabigatran accumulation. Dabigatran was studied in the context of total knee replacement in the RE-MODEL trial ( $n=2101$ patients $)$ and the RE-MOBILIZE trial $(n=2615$ patients) ${ }^{26}$ both of which were randomized, double-blind, double-dummy, active-control non-inferiority studies. Oral dabigatran $150 \mathrm{mg}$ or $220 \mathrm{mg}$ daily was compared with enoxaparin $40 \mathrm{mg}$ SC daily or $30 \mathrm{mg}$ SC twice daily. Primary efficacy end points included total VTE events and all-cause mortality. Dabigatran was no less effective than enoxaparin $40 \mathrm{mg}$ SC daily, but was inferior to enoxaparin $30 \mathrm{mg}$ SC twice daily.

Many factors must be taken into account if a patient's antiretroviral treatment is altered to accommodate other medical therapies. The aim of antiretroviral therapy continues to be maximal, lifelong, and continuous suppression of HIV replication to prevent emergence of resistance, to facilitate optimal immune recovery, and to improve health. The choice of regimen must consider convenience to the patient, tolerability, potential toxic effects, viral resistance, and drug interactions. In certain clinical scenarios, one or more agents in the regimen may be switched, as long as potency is maintained. ${ }^{27}$ Therapeutic drug monitoring is not recommended for general HIV care. However, it may be useful for pregnant women, children, and patients with renal or liver impairment, to minimize overexposure and adverse effects. ${ }^{27}$ In the case presented here, the patient was going to continue anticoagulation for a minimum of 6 months. He had had good virologic response to therapy with nevirapine, lamivudine, and zidovudine (defined as viral load less than 50 copies $/ \mathrm{mL}$ ), as well as reasonable tolerability. ${ }^{27}$ Given the anticipated short-term use of anticoagulation, it would not have been prudent to change his antiretroviral therapy.

One important aspect to consider in this case was the increased risk of thromboembolism in patients with HIV. The risk of VTE in the HIV population is approximately 2- to 10fold greater than in the general population. ${ }^{28}$ Risk factors having the strongest association with VTE include a diagnosis of AIDS and a low CD4+ count, especially in the presence of clinical AIDS, as well as protein $\mathrm{S}$ and protein $\mathrm{C}$ deficiencies, which are much more common in patients with HIV than in the general population. The question of whether certain risk factors (such as male sex, protease inhibitor therapy, presence of opportunistic infections [most commonly cytomegalovirus and Pneumocystis jiroveci], and presence of antiphospholipid antibodies) increase the risk of VTE in patients with HIV is controversial, as some evidence suggests a higher prevalence in HIV-infected individuals. ${ }^{28}$ Beyond being infected with HIV, the patient described here had no other risk factors for VTE. He had never been tested for protein $\mathrm{C}$ or protein $\mathrm{S}$ deficiency or the presence of antiphospholipid antibodies.

\section{CONCLUSIONS}

In the case reported here, a patient presented to hospital $24 \mathrm{~h}$ after discharge (following an admission for total knee replacement) with DVT and clinical signs of pulmonary embolism, specifically dyspnea, electrocardiographic changes, and elevated troponin T. Postoperatively and before the readmission, the patient had received a short course of dalteparin followed by 2 doses of rivaroxaban $10 \mathrm{mg}$ daily. Although the patient's HIV-positive status may have been a factor in the early presentation of VTE, concurrent nevirapine may have further increased the elevated hepatic clearance of rivaroxaban through induction of isozyme CYP3A4, leading to subtherapeutic anticoagulation. To the authors' knowledge, this is the first report of a potential drug interaction between nevirapine and rivaroxaban. Clinicians should consider the potential ramifications of enhanced clearance of rivaroxaban when this drug is used in conjunction with one or more enzyme-inducing agents.

\section{References}

1. Kwong LM, Tong LM. Drug interactions with rivaroxaban following total joint replacement surgery. Ann Pharmacother. 2012;46(9):1232-8.

2. Lassen MR, Ageno W, Borris LC, Lieberman JR, Rosencher N, Bandel TJ, et al.; RECORD3 Investigators. Rivaroxaban versus enoxaparin for thromboprophylaxis after total knee arthroplasty. $N$ Engl J Med. 2008;358(26):2776-86.

3. Turpie AGG, Lassen MR, Davidson BL, Bauer KA, Gent M, Kwong LM, et al.; RECORD4 Investigators. Rivaroxaban versus enoxaparin for thromboprophylaxis after total knee arthroplasty (RECORD4): a randomised trial. Lancet. 2009;373(9676):1673-80.

4. Melillo SN, Scanlon JV, Exter BP, Steinberg M, Jarvis CI. Rivaroxaban for thromboprophylaxis in patients undergoing major orthopedic surgery. Ann Pharmacother. 2010;44(6):1061-71.

5. Falck-Ytter Y, Francis CW, Johanson NA, Curley C, Dahl OE, Schulman $S$, et al. Prevention of VTE in orthopedic surgery patients: antithrombotic therapy and prevention of thrombosis, 9th ed: American College of Chest Physicians evidence-based clinical practice guidelines. Chest. 2012;141 (2 Suppl):e278S-e325S.

6. Facts and comparisons: eAnswers [database on Internet]. Indianapolis (IN): Wolters Kluwer Health, Inc; 2012 [cited 2012 Sep 21]. Available from: http://online.factsandcomparisons.com. Subscription required to access content. 
7. Micromedex $2.0{ }^{\circledR}$ healthcare series [database on intranet]. Version 5.1. Greenwood Village (CO): Thomson Reuters (Healthcare) Inc; 2012 [cited 2012 Sep 21]. Available from: www.thomsonhc.com. Subscription required to access content.

8. YouScript [personalized prescribing system on Internet]. Version 1.0. Seattle (WA): Genelex Corp; 2012 [cited 2012 Sep 21]. Available from: https://www.youscript.net/. Subscription required to access content.

9. Baxter K, editor. Stockley's drug interactions. 9th ed. London (UK): Royal Pharmaceutical Society; 2010.

10. Drug interaction tables. Toronto (ON): University Health Network, Toronto General Hospital, Immunodeficiency Clinic; 2012 [cited 2012 Sep 21]. Available from: www.hivclinic.ca/main/drugs_interact.html

11. McNicholl IR. Database of antiretroviral drug interactions [database on Internet]. San Francisco (CA): University of California, San Francisco, Center for HIV Information; 2012 [cited 2012 Sep 21]. Available from: http://hivinsite.ucsf.edu/InSite?page=ar-00-02

12. Lexicomp online [database on Internet]. Hudson $(\mathrm{OH})$ : Lexi-Comp, Inc; 2012 [cited 2012 Sep 18]. Available from: http://online.lexi.com. Subscription required to access content.

13. Xarelto [rivaroxaban] product monograph. Toronto $(\mathrm{ON})$ : Bayer Inc; 2012 Jul 18.

14. Drug interaction charts. Liverpool (UK): University of Liverpool; 2012 [cited 2012 Sep 21]. Available from: www.hiv-druginteractions.org

15. Horn JR, Hansten PD, Chan LN. Proposal for a new tool to evaluate drug interaction cases. Ann Pharmacother. 2007;41(4):674-80.

16. Wilkinson GR. Drug metabolism and variability among patients in drug response. N Engl J Med. 2005;352(21):2211-21.

17. Bates D, Burak KW, Coffin CS, Ying T, Enns EM. Phenytoin-induced reduction in sirolimus levels. Can J Hosp Pharm. 2011;64(4):271-4.

18. Wittkowsky AK. Novel oral anticoagulants and their role in clinical practice. Pharmacotherapy. 2011;31(12):1175-91.

19. Viramune [nevirapine] product monograph. Burlington (ON): Boehringer Ingelheim Canada; 2011 May 30.

20. Turpie AGG, Fisher WD, Bauer KA, Kwong LM, Irwin MW, Kalebo P, et al. BAY 59-7939: an oral, direct factor Xa inhibitor for the prevention of venous thromboembolism in patients after total knee replacement. A phase II dose-ranging study. J Thromb Haemost. 2005;3(11):2479-86.

21. Douxfils J, Mullier F, Loosen C, Chatelain C, Chatelain B, Dogné JM. Assessment of the impact of rivaroxaban on coagulation assays: laboratory recommendations for the monitoring of rivaroxaban and review of the literature. Thromb Res. 2012;130(6):956-66.

22. Asmis LM, Alberio L, Angelillo-Scherrer A, Korte W, Mendez A, Reber G, et al. Rivaroxaban: quantification by anti-FXa assay and influence on coagulation tests: a study in 9 Swiss laboratories. Thromb Res. 2012;129(4):492-8.
23. Mueck W, Lensing AWA, Agnelli G, Decousus H, Prandoni P, Misselwitz F. Rivaroxaban: population pharmacokinetic analyses in patients treated for acute deep-vein thrombosis and exposure simulations in patients with atrial fibrillation treated for stroke prevention. Clin Pharmacokinet. 2011;50(10):675-86.

24. Liedtke MD, Rathbun RC. Warfarin-antiretroviral interactions. Ann Pharmacother. 2009; $43(2): 322-8$.

25. Eliquis [apixaban] product monograph. Montreal (QC): Bristol-Myers Squibb Canada; 2012 Nov 27.

26. Burness CB, McKeage K. Dabigatran etexilate: a review of its use for the prevention of venous thromboembolism after total hip or knee replacement surgery. Drugs. 2012;72(7):963-86.

27. Thompson MA, Aberg JA, Hoy JF, Telenti A, Benson C, Cahn P, et al. Antiretroviral treatment of adult HIV infection: 2012 recommendations of the International Antiviral Society-USA panel. JAMA. 2012;308(4):387-402.

28. Kiser KL, Badowski ME. Risk factors for venous thromboembolism in patients with human immunodeficiency virus infection. Pharmacotherapy. 2010;30(12):1292-302.

Duane Bates, BScPharm, ACPR, is a Clinical Practice Leader, Medicine, in the Department of Pharmacy, Foothills Medical Centre, Alberta Health Services, Calgary, Alberta.

Bruce Dalton, BScPharm, PharmD, is a Clinical Practice Leader, Infectious Disease, in the Department of Pharmacy, Foothills Medical Centre, Alberta Health Services, Calgary, Alberta.

Janet Gilmour, MD, FRCPC, is Staff Physician in Internal Medicine, Department of Medicine, Foothills Medical Centre, Alberta Health Services, Calgary, Alberta.

Jeff Kapler, BScPharm, is a Clinical Pharmacist in the Southern Alberta HIV/AIDS Clinic, Alberta Health Services, Calgary, Alberta.

Competing interests: None declared.

\section{Address correspondence to:}

Duane Bates

Department of Pharmacy, Alberta Health Services

Foothills Medical Centre

1403 29th Street NW

Calgary AB T2N 2T9

e-mail: duane.bates@albertahealthservices.ca 